\title{
PLATELET PHAERESIS FOR COMPONENT THERAPY IN DHAKA MEDICAL COLLEGE- A STUDY OF 26 PROCEDURES
}

\author{
LAILA ARJUMAND BANU ${ }^{1}$, KAZI NOWSHAD HOSSAIN ${ }^{2}$
}

\begin{abstract}
:
Platelet separation by aphaeresis machine from single donor was started in Blood Transfusion Department of Dhaka Medical College Hospital, from 10th April 2002. This study includes 26 procedures done in this dept. from the period of 10 th April 2002 to 4th June 2003. Observation showed that demand of platelet came from different wards for different types of diseases such as , for Dengue- 8 cases, for Aplastic anaemia-2 cases, for Ovarian tumour-2 cases, for Caesarian section post operative DIC-2 cases, for AML with low platelet count-3 cases, for IUD with low platelet count - 1 case, for Blood cancer -1 case, for NHL chemotherapy induced thrombocytopenia1 case, for Dengue Shock Syndrome- 1 case, 5 units donated in Shishu Hospital. Platelet was supplied according to group specific of the patient. Such as, 12 cases were $O+v e, 10$ cases were $B$ $+v e, 3$ cases were $A+v e$ and 1 case was $A B+v e$.
\end{abstract}

\section{Introduction:}

Platelet can be seperated by cytaphaeresis or by separating platelet rich plasma from a unit of whole blood within $8 \mathrm{hr}$. of collection and recentrifuging to remove the majority of supernatant plasma. Platelets are suspended in a sufficient amount of plasma to ensure a $\mathrm{pH}$ of 6.0 or higher at the end of the allowable storage time.

One unit of platelet phaeresis (Single donor platelet) by aphaeresis machine contains a minimum of $3 \times 10^{11}$ platelets and is equivalent to 6 to 8 units of random donor platelets. ${ }^{1,2}$ Platelet concentration prepared from a single unit of whole blood (random donor platelet concentrate) usually contain a minimum 5.5 x $10^{10}$ platelets.

In a haematologically stable adult with $1.8 \mathrm{~m}^{2}$ body surface area, each unit of random donor platelet concentration is expected to increase the platelet count by approx (5 to 10$) \times 10^{8} / \mathrm{L}$. and one unit of platelets phaeresis increases the platelet count by (30 to 60) $\mathrm{x}$ $10^{9} / \mathrm{L}$ that is 6 times more yield then random one unit platelet concentration. Usual dose for thrombocytopenic bleeding adult is 6 to 10 random donor platelet concentration or one unit of platelets aphaeresis. $^{2}$

\section{Materials and Methods:}

Before doing the procedure the platelet donor was checked for - Physical fitness, Blood group was identical and compatible with the patients, Screening for Transfusion Transmissible infection was done, Platelet count was more than $2,50000 / \mathrm{cmm}$, Haematocrit (HCT) value was more than $36 \%$ and height, weight, pulse, blood pressure was within the acceptable range.

After donor selection, screening and completion of all the tests, donor is allowed to enter in the procedure room. Donor was lied down on the bed, I/V cannula was fitted on his right anticubital vein, normal saline drip started with the cannula on the right hand which will act as inlet site of anticoagulated blood from aphaeresis machine.

Patients were in comfort and relax position and patient was warmed with blanket. For platelet phaeresis special kit is required that is fitted with the machine. Machine was primed up with normal saline. Machine was programmed with the values of Haematocrit, haemoglobin, platelet count, height and Weight of the patients. After programming the machine gave the result of predicted yield of platelet, volume of platelet and the amount of anticoagulant used in the bag.

1. Associate Professor, Blood Transfusion Department, National Cancer Resarch Institute Hospital, Mohakhali, Dhaka.

2. Consultant, Transfusion Medicine Department, United Hospital, Dhaka. 
For outleting of blood left hand of the donor was used. In the left anticubital vein another needle was pricked and fixed in position to connect with the machine. When blood was going through the needle in the aphaeresis machine anticoagulant was mixed proportionality with blood before entering into the machine. After entry into the machine blood was centrifuged, in the interface where platelet remains in between red cell and plasma, platelets was separated and collected in another bag, rest of the blood was reinfused from machine to the donor by another tube to the needle through Right hand inlet site. Collected amount of platelet concentrate vary from 120 to 220 $\mathrm{ml}$ according to programming of the machine. After collection of platelet, the bag is leveled for - product name, date of collection patient group, expiry date, and kept in incubator at $20-22{ }^{\circ} \mathrm{c}$ with continuous horizontal agitation. In such way it can be kept for 2 to 5 days.

Sample of one procedure in which following documents were noted:

\begin{tabular}{ll}
\hline Donor No. - 26 & Date: 4-06-03 \\
Name - Abul Basher & Pulse - 100/ min \\
Age - 36 yrs & BP-130/80 mmHg \\
$\mathrm{Ht}-5^{\prime}-8^{\prime /}$ & Platelet count $-3,70000 / \mathrm{cmm}$ \\
$\mathrm{Wt}-73 \mathrm{~kg}$ & \\
$\mathrm{HCT}-42.9 \%$ & \\
\hline
\end{tabular}

TBV (Total Blood Value) - $4751 \mathrm{ml}$

\section{Machine result:}

Predicted yield of platelet $3.5 \times 10^{/ /}$concentration

Time - $100 \mathrm{~min}$ Volume - $260 \mathrm{ml}$

Actual yield of plateletTime - $60 \mathrm{~min}$ $2.7 \times 10^{/ /}$concentration Volume - $190 \mathrm{ml}$

Patient information:

Name - Kazal,

Age - 34 yrs

W - 21, B - 7, Reg no - 32853/39

Disease - Dengue shock syndrome

Blood group - $\mathrm{O}+$ ve

\section{Result:}

Out of 26 procedures of platelet phaeresis done in Blood Transfusion Department of Dhaka Medical College
Hospital, maximum platelet was used for Dengue patient- 8 cases, other causes of platelet use were Aplastic anaemia- 2 cases, Ovarian tumour- 2 cases post operative DIC after Caesarian section -2 cases, AML with low platelet count -3 cases, IUD with low platelet count - 1 case, Blood cancer - 1 case , chemotherapy induced thrombocytopenia in Non hodgkin lymphoma - 1 case, Dengue Shock Syndrome -1 case , 5 units donated in Shishu Hospital .

Platelet was supplied according to group specific for the patient. Such as, 12 cases were $\mathrm{O}+\mathrm{ve}, 10$ cases were $B+v e, 3$ cases were $A+v e$ and 1 case was $A B+v e$.

\section{Discussion: \\ $A B O$ and $R$ h type in platelet transfusion:}

$\mathrm{ABO}$ antigens are present on the surface of platelets and transfusion of $\mathrm{ABO}$ compatible platelets are required e.g. 'A' platelet to an 'O' recipient may have a slightly demised $24 \mathrm{hr}$ recovery. It is preferable to transfuse $\mathrm{ABO}$ compatible platelets. It is not necessary to crossmatch platelet concentrate with the recipient's red blood cells.

The $\mathrm{D}$ antigen is not present on the platelet surface. However $\mathrm{Rh}$ positive platelets containing $0.5 \mathrm{ml}$ or less of red blood cells carry a small risk of immunizing $\mathrm{Rh}$ negative individuals. It is therefore not desirable to transfuse $\mathrm{Rh}$ positive platelets to $\mathrm{Rh}$ negative femals of child bearing age. If this is unavoidable $\mathrm{Rh}$ immunoglobulin (Rh 1G) may be administered. Each $300 \mathrm{~mm}$ vial of $\mathrm{Rh} 1 \mathrm{G}$ provides protection against 15 $\mathrm{ml}$ of red cell present in 30 units $(0.5 \mathrm{ml}$ x 30 units $=15 \mathrm{ml}$ ) of $\mathrm{Rh}$ positive platelets.

Indication of platlets:

Many factors must be considered before initiating platelet therapy. The clinical condition of the patient, the number of functional ability of the patients own platelets, and cause of thrombocytopenia must all be evaluated. Patients with uncomplicated thrombocytopenia generally not require platelet transfusion until count fall below $10 \times 10^{9} / \mathrm{L}$. Serious complication (e.g. major gastrointestinal bleeding, Central Nervous system hemorrhage) do not arise until the platelet count is below $5 \times 10^{9} / \mathrm{L} .^{3,4}$

Most commonly platelets are transfused prophylactically to prevent spontaneous life threatening hemorrhage in individuals with transient thrombocytopenia due to treatment regimen for malignancy. ${ }^{3}$ 
By lowering the 'trigger value' for prophylactic platelet transfusion decreases the over all requirement for platelet transfusion and presumably diminishes the risk of alloimmmunization. The use of single donor rather than random donor platelets offers potential advantages to this population by limiting exposure to infectious agents and reducing the likelihood of alloimmunization5. During massive blood transfusion dilutional thrombocytopenia can result from multiple units of blood transfused to replace the lost through hemorrhage. Generally 35 to 40 percent of the patients own platelet remain following replacement of one blood volume. Following massive transfusion platelet should be given only when evidence is present of thrombocytopenia and clinically abnormal bleeding. ${ }^{5}$ It is important to remember that each unit of platelet concentrate contains approximately $50 \mathrm{ml}$ of relatively fresh plasma and according to the general guidelines for plasma therapy; this should provide $50 \mathrm{ml}$ of stable coagulation factors 3 . Patients with chronic thrombocytopenia caused by impaired platelet production (eg. aplastic anaemia) generally do not require prophylactic platelet transfusion. ${ }^{5}$

However, when thrombocytopenia and / or thrombocytopathy is present in a patient with uncontrollable blood loss (e.g. major surgery, gastrointestinal bleeding) platelets are indicated to help control bleeding and avoid life treating hemorrhage.

Alloimmunization:

Appropriate post transfusion monitoring is essential to determine the efficacy of the platelet transfusion. This is done by measuring the pre and post transfusion platelet count and noting the clinical effect of platelet transfusion (prevention or cessation of bleeding). An inadequate platelet response can be documented by comparing a corrected count increment (CCI) of the expected minimum response from the platelet transfusion. ${ }^{4}$

CCI $=($ Post - tx-plt ct. - pre- tx-plt ct.$) \times$ BSA Platelet transfusion $\mathrm{x} 10^{11}$

Where Post - tx-plt ct . = post transfusion platelet count

Pre tx plt ct $=$ Pre transfusion platelet count $\mathrm{BSA}=$ Body surface are in squire meter

Minimum acceptable CCI values-

$>7.5 \times 10^{9} / \mathrm{L}$ from a sample drown $10-60$

minutes, post transfusion

$>4.5 \times 10^{9} / \mathrm{L}$ from a sample drown $18-24$

hrs post transfusion

Fig.-1: $C C I$ - corrected count increment (CI)
The 10-60 minute CCI is considered to be diminished primarily by alloimmunization and splenomegaly, where as decrease in 18 to $24 \mathrm{hr}$ CCI is due to minimum cause such as fever, infection or DIC. ${ }^{2,6}$ When a patient become refractory due to alloimmunization, subsequent platelet transfusion from random donor fails to produce the expected therapeutic results. The majority of the platelets are immunized to HLA antigens. Leukocyte presents in routinely prepared platelet concentrate as well as the platelet themselves express HLA antigens that can provoke HLA antibody formation. A lymphocytotoxicity test (LCT) can be used to document the presence of LCT (HLA) antibodies percentage of reactive cells (cells injured by are antigen- antibody complement reaction that are detected by dye exclusion) correlates well with transfusion outcome. ${ }^{6}$

In order to improve the effectiveness of platelet transfusion in this alloimmunized population HLA matched platelet may be transfused. Reports have appeared recently in the literature of fatal transfusion associated graft-versus host disease (TA-GVHD) in recipient of cellular blood transfusion from closely related donors. ${ }^{6}$

The American Association of Blood Banks (AABB) has responded by recommending that blood and cellular products be irradiated with a minimum of $2500 \mathrm{CGY}$ (25 GY) in order to reduce the risk of TA-GVHD in bone marrow transplant recipient as well as recipients of donor units known to be from a blood relatives. Irradiation has had no adverse effects on the recovery survival and haemostatic efficacy of platelets following transfusion. ${ }^{7,8}$

Failure of HLA matched platelets to produce the expected past transfusion platelet count increment in all immunized patient suggests that the refractory state of the patient may be due to alloimmunization to platelet specific antigens. A platelet cross match can be used to detect antibodies to specific platelet antigen and thereby improve the responsiveness to platelet transfusion. ${ }^{6}$

\section{Conclusion:}

Platelet aphaeresis done in $\mathrm{DMCH}$ was mainly for patients suffering from Dengue, Dengue shock syndrome, Aplastic Anaemia, Acute myeloid leukemia, Blood cancer, DIC, IUD with low platelet count. By aphaeresis of platelet from single donor gives rich concentrate of platelet and prevents alloimmunization. 
Unit of issue -1 pack containing platelet concentration collected by cell separator device from a single donor -

Volume 150 - $300 \mathrm{ml}$

Platelet contain $150-500 \times 10^{9}$ equivalents to $3-10$ single donor

Storage, at $20{ }^{0} \mathrm{c}-24{ }^{0} \mathrm{c}$ up to $72 \mathrm{hr}$ with continuous agitation.

Dosage, is 1 unit of platelet concentration / $10 \mathrm{~kg}$ body wt. In a $70 \mathrm{~kg}$ adult 1 unit should raise the platelet count by $5000 /$ cumm. ${ }^{9}$

By this aphaeresis machine not only platelet aphaeresis can be done but other therapeutic aphaeresis -like plasma exchange, cytaphaeresis (removal of blood cell), progenitor cell (stem cell) collection can be done. For the procedure to be carried out a specialist team is essential, careful planning and coordination is needed for the preparation of the patient/ donor. It is a costly procedure no doubt but $\mathrm{Rh}$ negative mother with raised antibody titer and in other conditions where plasma exchange, stem cell collection is needed can be done with this machine within the country, instead of going abroad.

\section{References:}

1. Wedmann FK (ed). Standards for blood banks and transfusion services. 15 th ed. Arlington V, American Association of Blood Banks 1993.

2. Pisciotto PT (ed). Blood transfusion therapy a physicians Hand book. Arlington VA, American Association of Blood Bank 1989.

3. Walker RH (ed). Technical manual. Arlington VA, American Association of Blood Banks 1990.

4. Simpson MB. The clinical use of platelet preparation. In: Summer SH, Smith DM, Agranenko VA (eds). Transfusion therapy: Guide line for practice. Arlington, VA, American Association of Blood Banks 1990.

5. NIH consensus conference. Platelet transfusion therapy. JAMA 1987;257: 1777 - 80.

6. Evans CS. Alloimmunization and refractoriness to platelet transfusion. Lab Med 1992 ; 23:528 - 32 .

7. Murphy MF, water AH. Platelet transfusion: The problem of refractoriness. Blood Rev 1990; 4:16-24.

8. Buchholz DH, Miripol J, Aster RH, et al. Ultraviolet irradiation of platelets to prevent recipient alloimmunization (Abstracts 391). Transfusion 1988; 28:265.

9. The clinical use of Blood in Medicine, obstetrics, Paediatrics, Surgery and Anaesthesia, Trauma and Burns. WHO 2001; 87. 